15

\title{
Комбинированные электромагнитные подвесы с пониженным энергопотреблением для левитационного транспорта
}

\author{
() Е.Н. Андреев, ${ }^{1}$ Д.Н. Арсланова, ${ }^{1}$ Е.В. Ахметзянова, ${ }^{1}$ А.М. Базаров, ${ }^{1}$ В.Н. Васильев, ${ }^{1}$ О.С. Васильева, ${ }^{1}$ \\ М.С. Верхотуров, ${ }^{2}$ Е.И. Гапионок, ${ }^{1}$ А.А. Дёмина, ${ }^{1}$ С.В. Завадский, ${ }^{2}$ М.Ю. Зенкевич, ${ }^{3}$ М.В. Капаркова, ${ }^{1}$ \\ В.Д. Кузьменков, ${ }^{1}$ А.Н. Лабусов, ${ }^{1}$ М.С. Ларионов, ${ }^{1}$ М.В. Манзук, ${ }^{1}$ А.В. Мизинцев, ${ }^{4}$ А.Н. Неженцев, ${ }^{1}$ \\ Д.А. Овсянников, ${ }^{2}$ А.Д. Овсянников, ${ }^{2}$ М.В. Хохлов ${ }^{1}$
}

\author{
${ }^{1}$ Акционерное общество „НИИЭФА им. Д.В. Ефремова“, \\ 196641 Санкт-Петербург, Россия \\ ${ }^{2}$ Санкт-Петербургский государственный университет, \\ 199034 Санкт-Петербург, Россия \\ ${ }^{3}$ Академия материально-технического обеспечения им. генерала армии А.В. Хрулёва, \\ 199034 Санкт-Петербург, Россия \\ ${ }^{4}$ ООО „НИИЭФА-ЭНЕРГО“, \\ 196641 Санкт-Петербург, Россия \\ e-mail: sytch@sintez.niiefa.spb.su
}

Поступило в Редакцию 4 декабря 2018 г.

В окончательной редакции 4 декабря 2018 г.

Принято к публикации 21 декабря 2018 г.

\begin{abstract}
В настоящее время развитие магнитолевитационного транспорта ведется на базе электромагнитного и электродинамического подвесов, техническая и коммерческая реализация которых успешно продемонстрирована в Корее, Китае, Японии и других странах. Источниками электромагнитного поля в подвесах могут быть нормально проводящие электромагниты, сверхпроводниковые магниты, высококоэрцитивные постоянные магниты. Прогресс, достигнутый в разработке новых магнитных материалов (постоянные магниты и высокотемпературные сверхпроводники), открывает перспективы снижения энергопотребления левитационных транспортных систем. Авторами предложены магниты всех трех типов, которые совместно обеспечивают функционирование комбинированного электромагнитного подвеса, созданы масштабные макеты таких магнитов. Обеспечена перманентная левитация макетов подвеса с грузом. Подтверждена правильность технических решений, созданное в РФ программное обеспечение позволяет надежно масштабировать магнитные системы подвесов. Таким образом, имеются все предпосылки к дальнейшему этапу создания полномасштабных прототипов эффективных левитационных систем, в частности, грузовой платформы на 50 тонн.
\end{abstract}

DOI: 10.21883/JTF.2019.07.47811.419-18

\section{Введение}

Перспективные виды магнитолевитационного транспорта обычно ассоциируются с электромагнитным (EMS) или электродинамическим подвесами (EDS) [1-4]. Каждый из них обладает существенными достоинствами в соответствующих диапазонах скоростей движения транспортного средства и операционных режимах. Источниками электромагнитного поля в подвесах могут быть электромагниты (ЭМ), сверхпроводниковые (низкотемпературные и высокотемпературные) магниты, высококоэрцитивные постоянные магниты (ПМ). Существенный прогресс, достигнутый за последние годы в разработке новых магнитных материалов, позволяет рассмотреть возможность их комбинированного использования [5-7] для повышения эффективности и снижения энергопотребления транспортных систем.

В процессе анализа комбинированных подвесов был предложен ряд вариантов (патенты РФ № 2573524, № 2579446, № 2573135, № 2566507, № 2611858), которые различаются степенью интеграции элементов магнитных систем. В частности, рассматривались комбинации различных типов магнитов (источников поля) для одновременного применения в EMS. B качестве источников поля выступали нормально проводящие ЭМ, сверхпроводниковые (СП) магниты, (в том числе с использованием высокотемпературных сверхпроводников ВТСП-2), постоянные магниты на основе сплава $\mathrm{Nd}-\mathrm{Fe}-\mathrm{B}$.

К настоящему моменту времени разработаны варианты конструктивного исполнения магнитов всех упомянутых выше типов, и проведен анализ возможности серийного производства отдельных элементов магнитных систем. Разработаны детальные вычислительные модели, описывающие пространственное распределение электромагнитных полей, плотностей пондеромоторных сил (подъемной, тормозящей, опрокидывающей и стабилизирующей) и тепловыделений в проводящих элементах.

Разработаны и созданы натурные модели, подтверждающие правильность конструктивных решений. Выполнена верификация вычислительных моделей, методик и программного обеспечения, в частности, путем 
сопоставления с данными натурных экспериментов на разномасштабных моделях и результатами расчетов других авторов [8-11]. Проведен анализ возможности параметризации вычислительной модели с целью ее применения для систем, отличающихся значительным разбросом значений операционных параметров.

Показано, что созданная в России вычислительная технология [8-12] обеспечивает требуемую точность численного моделирования магнитных систем подвесов и может быть использована для решения задач анализа, синтеза и оптимизации таких систем. В общем виде эта технология базируется на решении стационарных и квазистационарных задач для нахождения пространственновременных распределений компонент электромагнитного поля, пондеромоторных сил и компонент других полей для обеспечения решения совместных задач.

Система уравнений макроскопического электромагнитного поля [13] в дифференциальной форме связывает векторы напряженностей электрического $\mathbf{E}$ и магнитного Н полей, векторы электрической $\mathbf{D}$ и магнитной $\mathbf{B}$ индукций между собой, а также с объемной плотностью свободных электрических зарядов $\rho$ и вектором объемной плотности электрического тока (тока проводимости) j.

В данном приложении помимо поля тока проводимости ј удобно принять существование в среде еще и заданного поля стороннего тока $\mathbf{j}^{e}$.

Система уравнений Максвелла, дополненная материальными уравнениями, законом Ома, граничными и начальными условиями, однозначно определяет решения всех задач макроскопической электродинамики. Для частных случаев эти уравнения упрощаются.

В случае квазистационарного приближения электромагнитное поле изменяется достаточно медленно, так что процессы поляризации успевают следовать за изменениями поля, и соотношения между рассматриваемыми пятью векторами D, E, B, H, j не зависят от производных этих векторов по времени. В силу конечной скорости распространения, поле переменных токов может удовлетворять условиям квазистационарности лишь в ограниченной области пространства [13]. Поскольку внутри металлов плотность токов смещения пренебрежимо мала по сравнению с плотностью проводимости для всех частот, применяемых в левитационных системах, первое уравнение Максвелла принимает вид $\nabla \times \mathbf{H}=\mathbf{j}+\mathbf{j}^{e}$, второе уравнение Максвелла для квазистационарных процессов записывают в форме $\nabla \times \mathbf{E}=\partial \mathbf{B} / \partial t$. (Здесь $\nabla$ - дифференциальный оператор Гамильтона, $t$ время, символ $\rho / \partial t$ означает дифференцирование по времени в данной точке пространства).

Для уменьшения размерности задачи весьма эффективным является известный подход, базирующийся на применении модифицированного или редуцированного или обобщенного скалярного магнитного потенциала, известного как „ $T-\Omega$ “-метод или метод векторного электрического потенциала в англоязычной литературе. В русскоязычной литературе используется определение „метод сведения вихревого магнитного поля к потенциальному полю источников“. В этом случае для искомого потенциала число неизвестных минимально и совпадает с числом узлов сетки.

Можно ввести вектор $\mathbf{P}$ такой, что $\nabla \times \mathbf{P}=\mathbf{j}$. Из условия $\nabla \times \mathbf{H}=\mathbf{j}$ может быть получено $\nabla \times \mathbf{H}=\nabla \times \mathbf{P}$ или $\nabla \times(\mathbf{H}-\mathbf{P})=0$. Соответственно для описания поля $\mathbf{H}-\mathbf{P}$ может быть введен скалярный „модифицированный“ или „обобщенный“ магнитный потенциал $\varphi$ : $\mathbf{H}-\mathbf{P}=-\nabla \varphi$ или $\mathbf{H}=-\nabla \varphi+\mathbf{P}$. Для известного вектора $\mathbf{P}$ распределение этого потенциала может быть найдено из решения краевой задачи для уравнения $\nabla \mu_{0} \mu(-\nabla \varphi+\mathbf{P})=0$, дополненного граничными условиями.

Вектор $\mathbf{P}$ может быть интерпретирован как удельный магнитный момент двойного магнитного слоя, который при известных условиях эквивалентен в магнитном отношении замкнутым токам [13]. Построение Р предполагает введение условных перегородок (магнитных листков), совпадающих в пространстве с двойными слоями. Эти перегородки должны опираться на замкнутые токовые контуры. Сама форма этих перегородок может быть произвольной. Вектор $\mathbf{P}$ в каждой точке совпадает по направлению с векторным элементом их поверхности. Выбор формы построения векторного электрического потенциала $\mathbf{P}$ определяется особенностями каждой конкретной задачи, принципами оптимизации алгоритма и возможностями программного обеспечения.

Данный подход отличается высокой эффективностью в случае электромагнитных систем, которые могут быть описаны моделью проводящих оболочек, и применим, если время диффузии магнитного поля $t$ через оболочку толщиной $h$ и проводимостью $\sigma=1 / \rho$ много меньше характерного времени $\Delta t$ анализируемого процесса, т.е. $\tau \approx \mu_{0} \sigma h^{2} \ll \Delta t$. В этом случае задача формулируется лишь относительно одной неизвестной - нормальной к оболочке компоненте векторного электрического потенциала $\mathbf{P}=P_{n} \mathbf{e}_{n}\left(\mathbf{e}_{n}-\right.$ орт нормали к оболочке $)$, определяемого соотношением $\mathbf{j} h=\nabla \times \mathbf{P}$, где $\mathbf{j}$ - плотность вихревого тока на оболочке.

Для односвязной оболочки $S^{\prime}$, находящейся во внешнем меняющемся поле с индукцией $\mathbf{B}_{\text {ext }}$, уравнение относительно $P_{n}$ может быть записано в виде [14]:

$$
\begin{aligned}
& e_{n}\left[\nabla_{r} \times \frac{\rho}{h}\left[\nabla_{r} P_{n}(t, \mathbf{r}) \times \mathbf{e}_{n}\right]\right]+\frac{\mu_{0}}{4 \pi} \\
& \times \frac{\partial}{\partial t} \int \frac{\left[\nabla_{r} P_{n}(t, \mathbf{r}) \times \mathbf{e}_{n}\right] \times\left(\mathbf{r}-\mathbf{r}^{\prime}\right)}{\left|\left(\mathbf{r}-\mathbf{r}^{\prime}\right)^{3}\right|} d S^{\prime}=-\frac{\partial B_{n, \text { ext }}(t, \mathbf{r})}{\partial t} .
\end{aligned}
$$

Однозначно определенный (с точностью до константы) потенциал $\mathbf{P}$ может быть введен только для односвязных поверхностей. В общем случае многосвязных и ветвящихся поверхностей для сведения их к односвязным подобластям вводятся условные разрезы и условные непроводящие поверхности.

Алгоритмы управления работой подвесов должны включать сценарии для номинальных, переходных и 


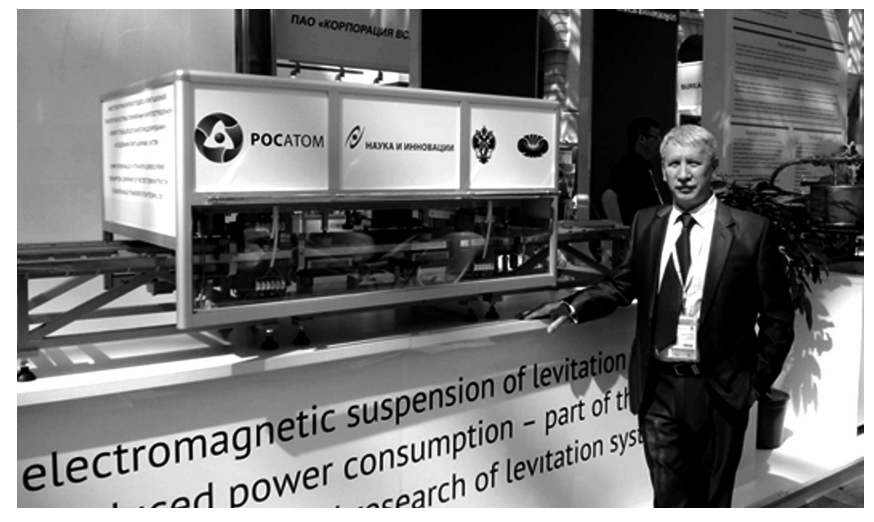

Рис. 1. Макет электромагнитного подвеса на выставке АТОМЭКСПО, Москва, 2017 г.

аварийных режимов работы. Практический опыт работы с макетами, обеспечивающими длительную устойчивую левитацию, показал, что построение таких алгоритмов управления [15] невозможно без применения детальных пространственных вычислительных моделей магнитных систем подвесов [16]. Такие модели должны включать детальное описание источников поля, токопроводящих элементов путевой структуры с учетом нелинейных магнитных свойств ферромагнитных элементов экипажа и путевой структуры.

Электромагнитный подвес (EMS) использует принцип активной магнитной опоры [1], который реализуется путем установки на транспортном средстве магнитов, испытывающих притяжение к ферромагнитным направляющим (рельсам). Для стабилизации подвеса используется активная система управления, которая регулирует величину магнитной индукции в левитационном зазоре, и, следовательно, силу притяжения магнитов $[1,16]$.

EMS используется как в статических режимах (погрузка, разгрузка, остановка), так и в режиме движения со скоростями менее $400-450 \mathrm{~km} / \mathrm{h}$, при которых EMS характеризуется сравнительно высокой (по-сравнению с EDS) энергоэффективностью. В рамках концепции EMS рассматривается магнитная система в виде комбинации источников поля:

- нормально проводящих ЭМ с активным управлениeм;

- постоянных магнитов для компенсации весовых нагрузок $[5,8]$;

- сверхпроводниковых ЭМ с „медленным“ изменением тока (компенсация поворотов, предварительная компенсация изменения веса при погрузке с возможным режимом перехода на использование ПМ).

Комбинированной левитационной системе присуща нелинейная зависимость подъемной и тормозящей составляющих сил от величин токов в магнитах, в том числе за счет наличия ферромагнитных элементов из электротехнических и конструкционных сталей.
Разработанные натурные макеты характеризуются близкими к реальным величиной левитационого зазора, быстродействием и точностью системы контроля и управления левитационным зазором, а также параметрами системы питания электромагнитов.

Основные задачи, которые решались в процессе работ на макетах, сводились к выбору оптимальных конфигураций ЭМ с целью минимизации энергетических затрат на обеспечение левитации, проверке общей концепции и элементов системы с магнитным подвесом на соответствие требованиям энергосбережения и экологической безопасности, оптимизации технологий изготовления системы магнитного подвешивания.

Цель настоящей работы состояла в разработке и наладке масштабного макета левитационной транспортной системы на основе комбинированного подвеса (КМП). На рис. 1 показан макет такой системы в процессе левитации, продемонстрированный в г. Москве на выставке АТОМЭКСПО в 2017 г. Макет используется в качестве элемента стенда для исследования алгоритмов управления левитационными системами.

\section{Левитирующий модуль и путевая структура}

Для всех элементов макета системы комбинированного подвеса, устанавливаемых на левитирующий модуль, была разработана конструкция несущей рамы (рис. 2). Ее изготовление, наряду с путевой структурой, позволили оценить требования к производству подобных систем.

В качестве основных параметров при разработке среднемасштабных макетов элементов EMS были приняты:

- левитационный зазор $5 \mathrm{~mm}$;

- расстояние между ферромагнитных направляющих $500 \mathrm{~mm}$;

- поперечный размер направляющих $25 \times 50 \mathrm{~mm}$;

- собственный вес левитирующего модуля не более $120 \mathrm{~kg}$;

- суммарная нагрузка на систему левитации не менее $250 \mathrm{~kg}$.

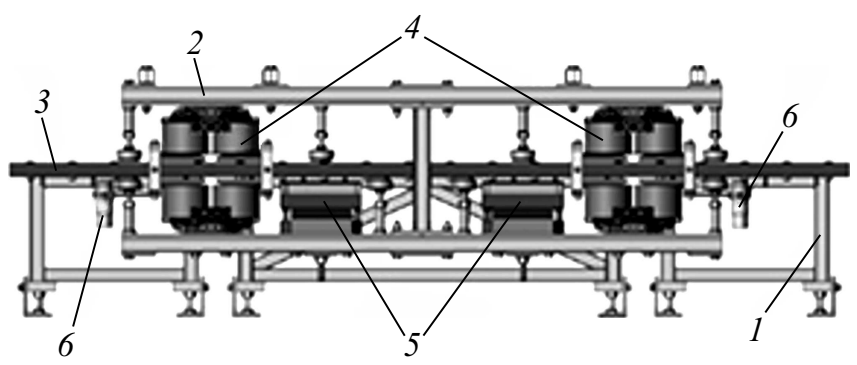

Рис. 2. Путевая структура и левитирующий модуль с установленными магнитами: 1 - путевая структура, 2 - левитирующий модуль, 3 - ферромагнитные направляющие, 4 - ЭМ, 5 - ОМ с ПМ, 6 - датчик измерения зазора. 


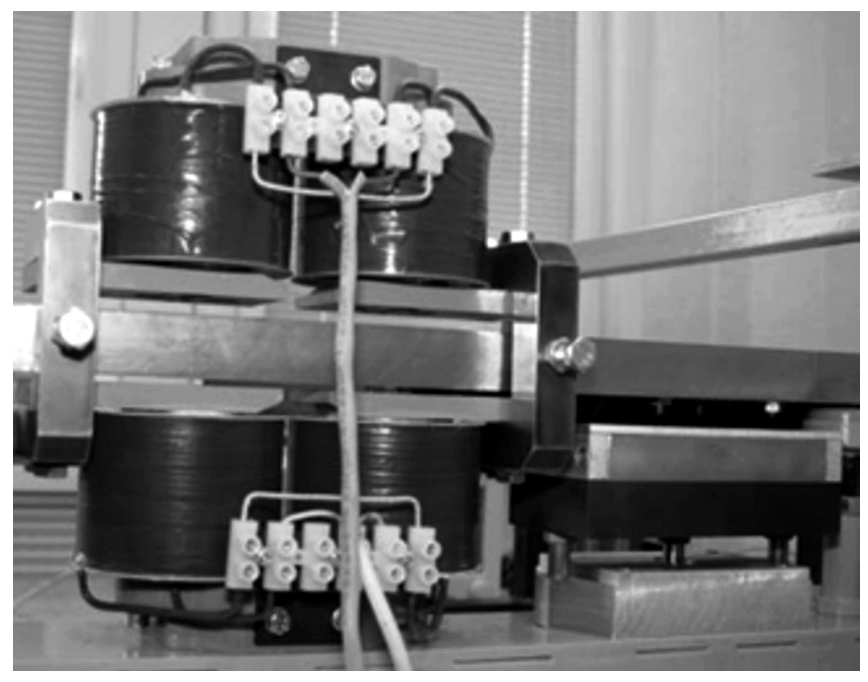

Pис. 3. Макеты ЭМ и ОМ с ПМ на стенде в режиме левитации.

Результаты численного моделирования пространственных распределений электромагнитных сил служили исходными данными для анализа напряженнодеформированного состояния системы „рама-путевая структура“. Была построена $3 D$-конечно-элементная модель, и проведены расчеты для различных условий работы и положений полезного груза (использовался комплекс программ ANSYS [17]), в частности, определены перемещения посадочных площадок несущей рамы для элементов магнитной системы. Конструкция рамы с учетом рекомендаций по результатам расчетов обеспечила требуемую степень жесткости, что подтверждено натурной моделью.

\section{EMS на базе нормально проводящих ЭМ}

Как известно [13,18], силы притяжения между стальными поверхностями могут быть сведены к силам натяжения, что указывает на качественную зависимость величины подъемной силы EMS от квадрата модуля индукции в левитационном зазоре и площади полюсов магнита. Проектирование и создание нормально проводящих ЭМ, входящих в состав макета, включали в себя следующие этапы: определение расчетным путем параметров магнита с учетом требуемой величины подъемной силы $\rightarrow$ конструкторская проработка $\rightarrow$ оптимизация конструкции $\rightarrow$ разработка документации $\rightarrow$ изготовление изделия $\rightarrow$ испытания (рис. 3). Расчеты распределений магнитных полей и сил проводились с использованием комплекса программ KOMPOT [12].

\section{EMS на базе комбинации нормально проводящих ЭМ и ПМ}

Была рассмотрена комбинированная магнитная система подвеса, в которой наряду с ЭМ используются ПМ (или/и СП-магниты). ПМ и СП-магниты обеспечивают основную грузонесущую функцию. Далее такие магниты будут называться опорными магнитами (OM). На первом этапе рассмотрены ОМ на базе ПМ (ОМ-ПМ). В этом случае основная функция нормально проводящих ЭМ состоят в поддержании устойчивой левитации. Эти электромагниты далее будут называться управляющими магнитами (УЭМ). Очевидно, что затраты на энергопотребление комбинированной системы подвеса будут значительно меньше, чем аналогичной по грузоподъемности системы EMS, использующей только ЭМ.

В рассматриваемом случае одной из основных проблем является разработка эффективных и устойчивых алгоритмов управления комбинированными системами, которые состоят из магнитов различных типов, расположенных, в общем случае, на различных расстояниях от ферромагнитных направляющих. Эти алгоритмы базируются на представлении функции управления в виде многомерной зависимости, включающей в себя величины левитационных зазоров, определяемых автоматической системой измерения для каждого УЭМ, и их производных. При этом влияние элементов магнитной системы учитывается соответствующими пространственными распределениями магнитных полей и сил.

Конструкция макета ОМ с ПМ приведена на рис. 4. Изготовленный ОМ с ПМ в составе левитирующего модуля показан на рис. 3.

Измерения величины силы притяжения ОМ к ферромагнитной направляющей $(\Phi Н)$ путевой структуры в зависимости от величины зазора между ними проводились на установке, состоящей из ряда устройств и средств измерения (рис. 5).

Блок ОМ-ПМ располагается над $Ф \mathrm{H}$ с зазором, закрепляется на коромысле и вывешивается на динамо-

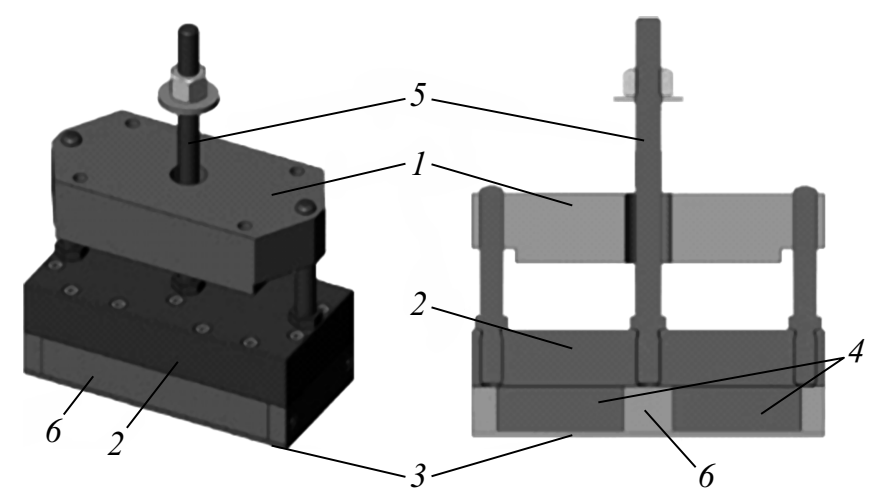

Рис. 4. ОМ с ПМ; слева - $3 D$-модель блока, справа - вид блока в разрезе: 1 - узел крепления, 2 - стальной магнитопровод, 3 - нижняя пластина кормуса ПМ, 4 - сборка ПМ, 5 - шпилька подвеса блока, 6 - корпус ПМ. 


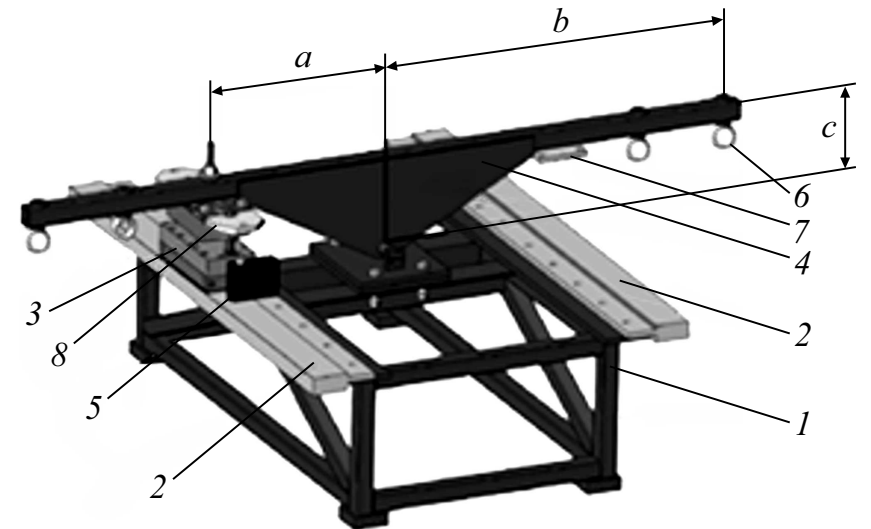

Рис. 5. Общий вид несущего каркаса с элементами путевой структуры и установкой для измерения величины силы притяжения ОМ: 1 - каркас, $2-\Phi \mathrm{H}, 3-\mathrm{OM} \mathrm{c} \mathrm{ПМ,}$ 4 - коромысло, 5 - лазерный датчик, 6 - крепление для груза, 7 - пластина крепления ЭМ, 8 - узел крепления ОМ.

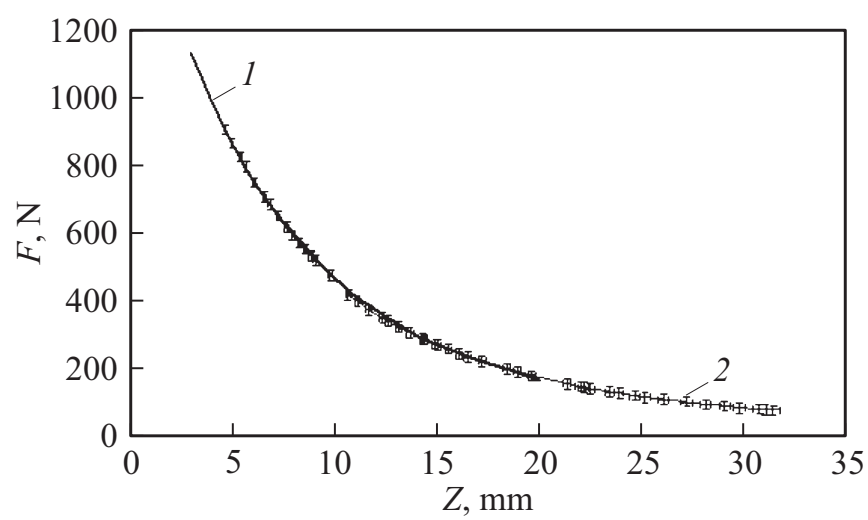

Рис. 6. Расчетные (1) и измеренные (2) с учетом приведенной погрешности измерений силы притяжения ОМ с ПМ к ферромагнитной направляющей путевой структуры.

метре с помощью талрепа, закрепленного на вспомогательном каркасе.

Сила притяжения блока ОМ-ПМ к ФН измеряется непосредственно динамометром, имеющим погрешность, не превышающую $\pm 10 \mathrm{~g}$ во всем диапазоне измерения силы. Поскольку при малых зазорах сила притяжения превышает предел измерения динамометра, то часть этой силы уравновешивается дополнительным грузом на противоположном плече коромысла.

Зазор между ОМ-ПМ и ФН регулируется с помощью талрепа, а величина зазора измеряется лазерным триангуляционным датчиком РФ603-55/30, имеющим погрешность измерений не более $\pm 5 \mu \mathrm{m}$.

Совпадение с требуемой точностью результатов измерений и численного моделирования (рис. 6) позволяет верифицировать расчетную модель ОМ-ПМ.

Аналогичные измерения были выполнены для нормально проводящих УЭМ. Сравнение результатов моделирования и натурных измерений также продемонстри- ровало приемлемую точность разработанной вычислительной модели.

Вычислительные модели УЭМ и ОМ-ПМ были использованы при разработке алгоритмов управления положением левитирующего модуля относительно ферромагнитных направляющих путевой структуры. Макет комбинированного подвеса с магнитной системой, содержащей группы управляющих ЭМ и ПМ (рис. 1), продемонстрировал устойчивую левитацию и требуемую грузоподъемность. Выполненные экспериментальные работы с макетом подтвердили работоспособность предложенной концепции комбинированного магнитного подвеса.

\section{EMS на основе СП ЭМ}

При применении в системе комбинированного подвеса СП-магнитов (в частности, на основе ВТСП-2 технологий) можно ожидать существенного повышения ее эффективности за счет снижения энергозатрат и повышения уровня рабочего поля. Проектирование и создание СП-магнитов проводились с учетом требований к функционированию макета и опыта работы в области прикладной сверхпроводимости (рис. 7). Испытания СП-магнитов и экспериментальные исследования их грузоподъемности подтвердили расчетные параметры разработанных магнитов.

\section{Результаты испытаний потребляемой мощности макета EMS}

Эксперименты, проведенные на макете EMS, показали, что ОМ-ПМ могут компенсировать значительную часть веса транспортной платформы. Таким образом, достигается экономия электроэнергии по сравнению c „классическим“ EMS. Устойчивая левитация макета, обеспечиваемая динамическим регулированием поля УЭМ с использованием обратной связи датчиков зазора, демонстрирует работоспособность разработанного комбинированного подвеса.

Следует отметить, что одновременно с ПМ в качестве опорных могут применяться и СП-магниты. Одновременное использование трех типов источников магнитно-

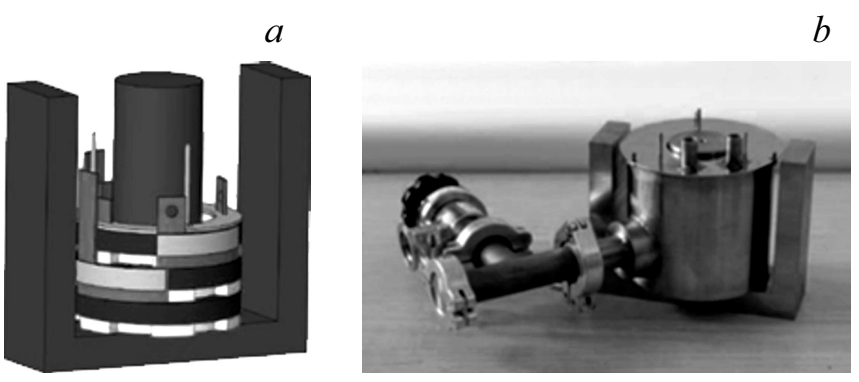

Рис. 7. CAD-модель $(a)$ и натурный макет СП-магнита с криостатом $(b)$. 


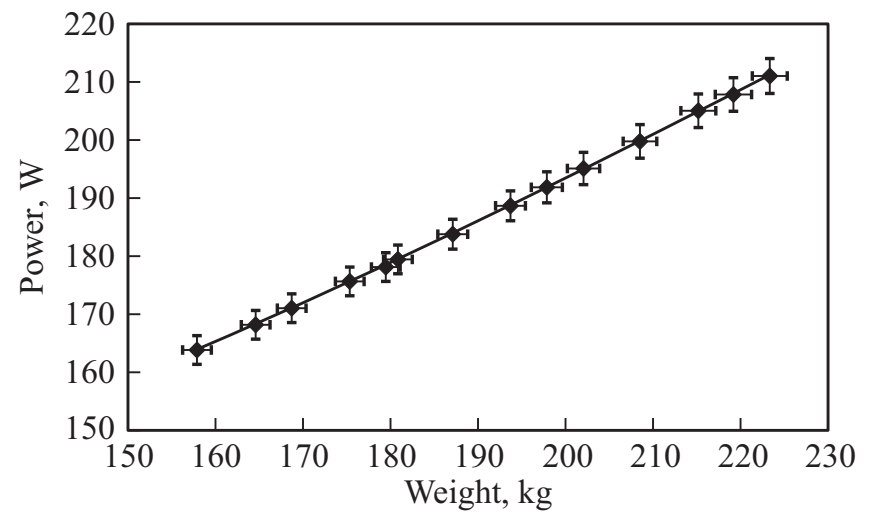

Рис. 8. Зависимость мощности затрат от веса левитирующего модуля макета.

го поля делает систему более гибкой в плане управления грузоподъемностью подвеса. Использование высокотемпературных сверхпроводников, способных работать при температуре жидкого азота, также существенно уменьшает затраты на электроэнергию для обеспечения необходимой подъемной силы.

Проведены измерения зависимости потребляемой мощности УЭМ от веса груза, при этом авторы не ставили перед собой задачу оптимизации системы на данном этапе макетирования. Получена характерная оценка величины мощности затрат $0.75 \mathrm{~W} / \mathrm{kg}$ при фиксированном положении ОМ-ПМ и левитационном зазоре $4.5 \mathrm{~mm}$ между путевой структурой и полюсами УЭМ (рис. 8). При этом подъемная сила, обеспечиваемая OM, составляла $135 \mathrm{~kg}$. С уменьшением левитационного зазора потребляемая мощность уменьшается.

Интересно отметить, что полученная оценка удельной мощности сопоставима с затратами мощности на преодоление сил трения качения в подшипниках колес и колес о рельсы для традиционного подвеса „колесорельс“" поездов высокоскоростных магистралей [19].

Существует возможность дальнейшего увеличения подъемной силы при той же мощности затрат путем регулировки вертикального положения ОМ-ПМ. Подобная регулировка может быть осуществлена за счет применения дополнительных управляющих механизмов. Гораздо целесообразнее в этом плане выглядит использование сверхпроводниковых ОМ.

\section{Заключение}

Работоспособность концепции комбинированного электромагнитного подвеса левитационного транспорта с пониженным энергопотреблением подтверждена результатами численного и натурного моделирования.

Оптимизация конструкции и режимов работы комбинированного подвеса требует расчетов с использованием $3 D$-детальных моделей. Существующая вычислительная технология анализа, синтеза и оптимизации магнитных систем электрофизических установок обеспечивает для магнитных систем подвесов надежное масштабирование их параметров в требуемом диапазоне их изменения.

Численное моделирование дополнено экспериментами с использованием натурных моделей различного масштаба. Работа с макетами, в частности, обеспечила верификацию вычислительных моделей магнитной системы подвесов. Это позволило разработать и испытать на макетах алгоритмы управления левитирующим модулем в близких к практическим условиях его работы.

\section{Список литературы}

[1] Журавлев Ю.Н. Активные магнитные подшипники. Теория, расчет, применение. СПб: Политехника, 2003. 206 с.

[2] Транспорт с магнитным подвесом / Под ред. В.И. Бочарова, В.Д. Нагорского. М.: Машиностроение, 1991. 320 с.

[3] Дзензерский В.А., Омельяненко В.И., Васильев С.В., Матин В.И., Сергеев С.A. Высокоскоростной магнитный транспорт с электродинамической левитацией. Киев: Наукова думка, 2001. $480 \mathrm{c}$.

[4] Ким К.К. Системы электродвижения с использованием магнитного подвеса и сверхпроводимости. М.: ГОУ „Учебно-методический центр по образованию на железнодорожном транспорте“, 2007. 360 с.

[5] Kim Y., Kim K., Lee J. // IEEE Tr. Magn. 2001. Vol. 37. N 4. P. 116-132.

[6] Chen Q., Li J., Li G., Zhou S. // The $3^{\text {rd }}$ International Conference on Mechatronics and Mechanical Engineering (ICMME 2016), MATEC Web Conferences, 2017. Vol. 95. P. 5.

[7] Kireev A.V., Kozhemyaka N.M., Kononov G.N. // Int. J. Appl. Eng. Res. 2017. Vol. 12. N 18. P. 7219-7224.

[8] Амосков В.М., Арсланова Д.Н., Базаров А.М., Белов А.В., Беляков В.А., Белякова Т.Ф., Васильев В.Н., Гапионок Е.И., Зайцев А.А., Капаркова М.В., Кухтин В.П., Ламзин Е.А., Ларионов М.С., Максименкова Н.А., Михайлов В.М., Нежсенцев А.Н., Овсянников Д.А., Овсянников А.Д., Родин И.Ю., Сычевский С.Е., Фирсов А.А., Шатиль Н.А. // Вестник Санкт-Петербургского ун-та. 2014. Сер. 10. Вып. 4. С. 5-15.

[9] Амосков В.М., Арсланова Д.Н., Базаров А.М., Белов А.В., Беляков В.А., Белякова Т.Ф., Васильев В.Н., Гапионок Е.И., Зайцев А.А., Капаркова М.В., Кухтин В.П., Ламзин Е.А., Ларионов М.С., Максименкова Н.А., Михайлов В.М., Нежсенцев А.Н., Овсянников Д.А., Овсянников А.Д., Родин И.Ю., Сычевский С.Е., Фирсов А.А., Шатиль Н.А. // Вестник Санкт-Петербургского ун-та. 2015. Сер. 10. Вып. 2. С. 17-31.

[10] Амосков В.М., Арсланова Д.Н., Базаров А.М., Белов А.В., Беляков В.А., Белякова Т.Ф., Васильев В.Н., Гапионок Е.И., Зайцев А.А., Капаркова М.В., Кухтин В.П., Ламзин Е.А., Ларионов М.С., Максименкова Н.А., Михайлов В.М., Неженцев А.Н., Овсянников Д.А., Овсянников А.Д., Родин И.Ю., Сычевский С.Е., Фирсов А.А., Шатиль Н.А. // Вестник Санкт-Петербургского ун-та. 2015. Сер. 10. Вып. 3. С. 4-20.

[11] Амосков В.М., Арсланова Д.Н., Базаров А.М., Белов А.В., Беляков В.А., Белякова Т.Ф., Васильев В.Н., Гапионок Е.И., Зайцев А.А., Зенкевич М.Ю., Капаркова М.В., Кухтин В.П., Ламзин Е.А., Ларионов М.С., Максименкова Н.А., Михайлов В.М., Нежсенцев А.Н., Овсянников Д.А., 
Овсянников А.Д., Родин И.Ю., Сычевский С.Е., Фирсов А.А., Шатиль Н.А. // Вестник Санкт-Петербургского ун-та. 2016. Сер. 11. Вып. 3. С. 4-17.

[12] Belov A.V., Belyakova T.F., Gornikel I.V., Kukhtin V.P., Kuchinsky V.G., Lamzin E.A., Semchenkov A.G., Shatil N.A., Sytchevsky S.E. // IEEE Tr. Appl. Supercon. 2008. Vol. 18. N 2. P. $1609-1612$.

[13] Тамм И.Е. Основы теории электричества. М.: ГИТТЛ, 1954. $620 \mathrm{c}$.

[14] Belov A.V., Doinikov N.I., Duke A.E., Kokotkov V.V., Korolkov M.D., Kotov V.L., Kukhtin V.P., Lamzin E.A., Sytchevsky S.E. // Fusion Eng. Des. 1996. Vol. 31. P. $167-180$.

[15] Завадский С.В., Шароватова Д.С. // Процессы управления и устойчивость. 2015. Т. 2. № 1. С. 409-415.

[16] Amoskov V., Arslanova D., Baranov G., Bazarov A., Belyakov V., Firsov A., Kaparkova M., Kavin A., Khokhlov M., Kukhtin V., Kuzmenkov V., Labusov A., Lamzin E., Lantzetov A., Larionov M., Nezhentzev A., Ovsyannikov D., Ovsyannikov A., Rodin I., Shatil N., Sytchevsky S., Vasiliev V., Zapretilina E., Zenkevich M. // Cybernet. Phys. 2018. Vol. 7. N 1. P. $11-17$.

[17] Программные продукты ANSYS [Электронный ресурс] // www.cadfem-cis.ru: Кадфем Россия. Режим доступа: http://www.cadfem-cis.ru/products/ansys, свободный.

[18] Попов В.С., Мансуров Н.Н., Николаев С.А. Электротехника. М.: Из-во мин. коммун. хоз-ва РСФСР, 1956. 351 с.

[19] Кирякин В.Ю., Лежава В.Ш., Новгородцева А.В., Соколова М.В., Понарин Л.Н. // Бюлл. объединенного ученого совета ОАО „РЖД“. 2016. № 5. С. 28-36. 